\title{
Relação entre flexibilidade e força muscular em adultos jovens de ambos os sexos
}

\author{
Ana Cristina Gouvêa Carvalho ${ }^{1}$, Karla Campos de Paula ${ }^{2}$, \\ Tânia Maria Cordeiro de Azevedo ${ }^{2}$ e Antonio Claudio Lucas da Nóbrega ${ }^{3}$
}

\section{RESUMO}

Padrões adequados de força muscular e flexibilidade permitem movimentação eficiente, melhorando a performance desportiva e conferindo qualidade de vida. Entretanto, não se conhece a potencial interferência mútua entre força e mobilidade articular. $\mathrm{O}$ objetivo deste estudo foi investigar a relação entre força muscular e flexibilidade global e segmentar em adultos jovens. Cinqüenta (30h; 20m; idade $22 \pm 4$ anos) indivíduos sadios foram submetidos a avaliação cineantropométrica (peso corporal, altura, circunferências, dobras cutâneas), de mobilidade articular máxima passiva (método Flexiteste, que compara a amplitude atingida com mapas de referência) e de força muscular esquelética máxima [método de uma repetição máxima (1RM) de handgrip, legpress e supino horizontal]. Os resultados de força muscular foram corrigidos pela circunferência muscular correspondente, calculada como circunferência do membro subtraída da dobra cutânea vezes valor de $\pi$. A flexibilidade global (flexíndice = somatório dos resultados dos 20 movimentos articulares) foi maior nas mulheres [(mediana e amplitude) $=52$ (3869)] em relação aos homens [46 (37-57); $\mathrm{p}=0,004]$ à custa de maior flexibilidade segmentar (somatório dos resultados de movimentos relacionados) de quadril $(\mathrm{p}=0,004)$, coluna $(\mathrm{p}=0,006)$ e membros inferiores $(\mathrm{p}=0,011)$, enquanto a força muscular global e por movimentos foi superior nos homens $(\mathrm{p}=0,001)$. Não existiu correlação entre flexibilidade e força muscular para mulheres ou homens, seja do ponto de vista global (mulheres: $\mathrm{r}=0,149 ; \mathrm{p}=0,531$; homens: $\mathrm{r}=$ $0,092 ; p=0,628)$ ou segmentar $(p>0,05)$. Concluímos que, considerando a faixa etária estudada, as mulheres têm maior

1. Bolsista de iniciação científica do CNPq.

2. Professora Auxiliar do Departamento de Educação Física da Universidade Federal Fluminense, Niterói, RJ.

3. Professor Adjunto; Chefe do Departamento de Fisiologia da Universidade Federal Fluminense, Niterói, RJ.

Endereço para correspondência:

Rua Presidente Pedreira, 35/1.302, bl. 1, Ingá

24210-470 - Niterói, RJ

Tel: (021) 620-1789 / Fax: (021) 714-6821

E-mail: anobrega@web4u.com.br flexibilidade, principalmente nos movimentos de coluna, quadril e membros inferiores, enquanto os homens apresentam maior força muscular global e segmentar, mesmo corrigindo-se a diferença de massa muscular. Os resultados sugerem que não existe relação entre força muscular e flexibilidade em adultos jovens sadios.

Palavras-chave: Flexibilidade. Força muscular. Flexiteste.

\section{ABSTRACT \\ Relationship between muscular strength and flexibility in healthy adults of both genders}

Adequate levels of muscle strength and flexibility allow for efficient movements, improving sports performance and providing a better quality of life. However, the potential mutual interference between strength and joint motion is unknown. The purpose of the study was to investigate the relationship between global and regional muscle strength and flexibility in young adults. Fifty (30 men, 20 women, age 22 \pm 4 years) healthy subjects were submitted to an evaluation consisting of kineanthropometry (body weight, height, limb girths, skinfolds), measurement of the maximal passive range of joint motion (flexitest method which compares the joint range achieved to reference maps) and of the peak skeletal muscle strength [one-repetition maximum (1-MR) test of handgrip, legpress and bench press]. The results of muscle strength were corrected by the corresponding muscular girth, calculated by subtracting the limb girth from the skinfold times the $\pi$ value. The global flexibility (the sum of the result of all 20 joint movements) was higher in women [(median and range $=52(38-69)]$ compared to men [46(37-57); $p=0.004]$ due to a higher regional flexibility (the sum of related movements) of the hip ( $p=0.004)$, spine $(p=0.006)$ and lower limbs $(p=0.011)$, whereas the strength of each movement was higher in men $(p=0.001)$. There was no correlation between flexibility and muscle strength either for men or women, when all data points were pooled (women: $r=0.149 ; p=$ 0.531; men: $r=0.092 ; p=0.628$ ) or separated by body regions ( $p>0.05)$. The authors concluded that, considering the age range studied, women presented higher flexibility than men, particularly for the movements of the spine, hip 
and lower limbs, while men present higher global and regional muscle strength, even when corrected for the difference in muscle mass. Results suggest that there was no relationship between muscle strength and flexibility in healthy young adults.

Key words: Flexibility. Flexitest. Muscle strength.

\section{INTRODUÇÃO}

Níveis adequados de força muscular e mobilidade articular contribuem para a execução de movimentos eficientes e manutenção do equilíbrio, correlacionando-se positivamente com a qualidade de vida ${ }^{1-5}$. Por outro lado, o declínio da flexibilidade e da performance muscular que ocorre ao longo dos anos correlaciona-se com a diminuição da autonomia e a capacidade para realizar atividades cotidianas nos indivíduos idosos ${ }^{6-8}$.

Diferentes mecanismos explicam a relação entre flexibilidade e qualidade de vida. Por exemplo, movimentos envolvendo articulações com flexibilidade limitada são executados com menor eficiência mecânica e, portanto, maior gasto energético ${ }^{3}$. Além disso, a menor flexibilidade das regiões lombar e posterior da coxa parece predispor ao desenvolvimento de lombalgia crônica ${ }^{8}$. Ao lado da flexibilidade, a performance muscular também está envolvida na qualidade de vida. Toda atividade física, incluindo as do cotidiano, exige o envolvimento de certo percentual da força e endurance máximos dos indivíduos ${ }^{1,9}$. Por essa razão, o ganho da força muscular máxima faz com que as mesmas atividades representem menor carga relativa e, conseqüentemente, menor estresse fisiológico. Outro aspecto importante diz respeito ao efeito de aumento da massa óssea e da resistência do tecido conectivo como adaptação benéfica ao treinamento de força $^{1-5}$. Considerando-se esse mecanismo, o treinamento contra resistência torna-se importante, tanto na adolescência ${ }^{10}$, pois favorece o desenvolvimento de massa óssea total maior, quanto em indivíduos idosos ${ }^{8}$, particularmente em mulheres pós-menopausa, que passam a perder densidade óssea mais aceleradamente ${ }^{5}$.

A flexibilidade e a força muscular são qualidades físicas importantes não só para a promoção da saúde, mas também para a performance no esporte competitivo. Os diferentes esportes dependem do desenvolvimento em graus variados da proporção entre as diferentes qualidades físicas. Em certas modalidades como, por exemplo, nos arremessos e nas lutas, a força muscular é fundamental, enquanto a mobilidade articular mostra-se capital para a qualidade da performance na ginástica e no nado sincronizado ${ }^{11,12}$.

Apesar da sólida base teórica sobre a importância isolada da flexibilidade e da força muscular, ainda permanece obscura a relação entre estas duas qualidades físicas ${ }^{13}$. Assim, justifica-se a realização de outros estudos envolvendo a avaliação concomitante da flexibilidade e da força muscular de diferentes segmentos e articulações. O objetivo deste estudo foi determinar, em indivíduos adultos jovens saudáveis, a relação entre força muscular e flexibilidade global e segmentar.

\section{METODOLOGIA}

\section{Amostra}

Um total de 50 alunos de graduação de diferentes cursos [20 mulheres, 30 homens; idade (média \pm DP): $21 \pm 4$ anos], inscritos na disciplina de Educação Física da Universidade Federal Fluminense, concordaram em participar, assinando um termo de consentimento após esclarecimento sobre os objetivos, protocolo e riscos do estudo.

Todos os voluntários responderam a um questionário de prontidão para a atividade física (PAR-Q) $)^{14}$ com objetivo de identificar possíveis contra-indicações ao teste de flexibilidade e de força máxima. Uma anamnese médica direcionada dos voluntários identificados na avaliação inicial através do PAR-Q foi realizada por um dos autores.

\section{Protocolo}

Todos os voluntários foram submetidos à antropometria ${ }^{15}$, medida da flexibilidade articular e da força muscular esquelética. Os dados foram obtidos no mesmo dia, sempre na seqüência: antropometria, flexibilidade e força muscular.

\section{Antropometria}

Foram obtidas as circunferências de braço, antebraço, coxa e perna (panturrilha) com uma fita métrica não elástica. Dobras cutâneas de pontos coincidentes com as medidas da circunferência foram obtidos com um compasso de dobras cutâneas (Harpender, Inglaterra). A circunferência muscular foi calculada pela circunferência do membro subtraído da dobra cutânea relacionada multiplicada por $3,1416(\pi)^{16}$. Todas as medidas foram obtidas no lado direito, segundo padronização internacional ${ }^{17}$. Altura e peso também foram registrados antes e após o período de treinamento.

\section{Medida da flexibilidade}

A amplitude máxima passiva de 20 movimentos articulares em ombro, membro superior, quadril, membro inferior e tronco foi medida através do Flexiteste ${ }^{18}$. Nesse método, atribui-se a cada movimento articular um valor inteiro de 0 a 4 , comparando-se a posição obtida com as do mapa de referência (anexo 1). Nesse método padronizou-se que os movimentos são medidos do lado direito sem aquecimento prévio. A fidedignidade do Flexiteste foi determinada anteriormente, obtendo-se coeficientes de correlação intraclasse médios de 0,94 e 0,89 para a fidedignidade intra e interobservadores, respectivamente. Vale notar que coeficientes de correlação intraclasse superiores a 0,75 são considerados excelentes índices de fidedignidade ${ }^{19}$. O somatório dos valores medidos em todos os movimentos corresponde ao flexíndice, que foi utilizado para quantificar a flexibilidade global. A partir dos resultados dos movimentos individuais, foram calculados 


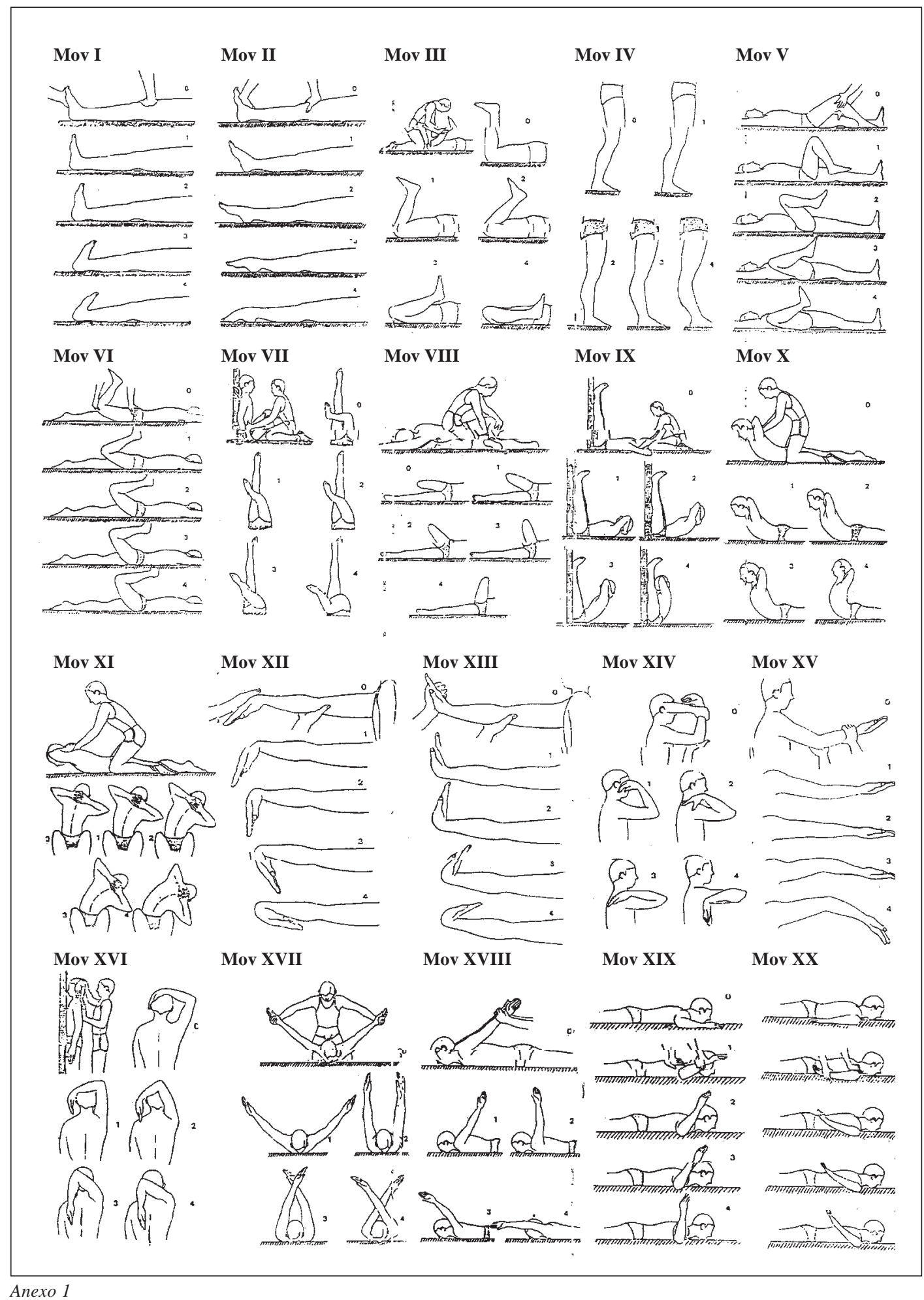

índices de flexibilidade segmentar, somando-se os movimentos relacionados a cada articulação (anexo 1): ombro (movimentos de XVI a XX), quadril (V a VIII), coluna (IX a XI), membro inferior (I a IV) e membro superior (XII a XV).
Apresentamos a descrição por escrito de apenas alguns movimentos do Flexiteste como exemplo. As posições do avaliador e do avaliado nos movimentos podem ser observadas no mapa de referência (anexo 1). 


\section{ANEXO 1}

Movimento I - flexão dorsal do tornozelo - Deve-se eliminar qualquer tensão muscular contrária ao movimento por parte do avaliado; um ângulo reto entre o pé e a perna ainda corresponde ao valor de 1; é comum levantar um pouco o calcanhar do solo na execução do movimento e isto não interfere com a avaliação; o avaliador observa pela face interna do pé do avaliado.

Movimento V - flexão do quadril - Em alguns casos, é necessário que o avaliador utilize o peso do seu corpo para conseguir a amplitude passiva máxima no movimento, usando para isso as duas mãos sobre a perna direita do avaliado e seu joelho direito para manter a perna esquerda do avaliado estendida; para alcançar as amplitudes correspondentes aos valores 3 e 4 é necessário executar pequena abdução do quadril avaliado; é muito importante evitar que haja rotação do quadril, o que pode ser detectado pela perda de contato entre a nádega esquerda e o solo.

Movimento IX - flexão do tronco - É conveniente que o avaliado inicie o movimento, de modo a diminuir o emprego da força por parte do avaliador; também é melhor para o avaliador colocar suas mãos supinadas na região das escápulas e no oco axilar do avaliado; é extremamente importante encostar bem as nádegas na parede, assim como evitar a flexão dos joelhos; quando somente se descola do solo a coluna cervical temos o valor 1 , enquanto a mesma situação para a coluna lombar corresponde a 3 e com superposição completa do tórax e parte anterior das coxas é atribuído o valor 4; no caso em que o avaliado não consegue sequer assumir a posição para a realização do movimento, consigna-se o valor 0 .

Movimento XII - flexão do punho - É importante não permitir a flexão do cotovelo para um julgamento correto; não se deve exercer pressão sobre os dedos e sim na região metacarpiana; na realidade, os dedos não devem ser levados em consideração para a avaliação; o braço do avaliado está estendido à frente do corpo sem qualquer abdução do ombro correspondente; o avaliador observa o movimento pelo lado interno do braço do avaliado.

Movimento XVII - extensão +adução posterior ou extensão posterior do ombro - Quando existe ângulo de 90 graus entre os braços e o corpo do avaliado, temos o valor 2; quando existe superposição dos punhos, o valor atribuído é 3 , sendo de 4 o valor medido quando se verifica superposição dos cotovelos.

\section{Medida da força muscular}

A força muscular máxima foi medida pelo método de uma repetição máxima (1RM) para legpress e desenvolvimento supino horizontal e pela dinamometria para preensão manual estática direita (handgrip) ${ }^{1-4}$. Alguns minutos de aquecimento e familiarização com os equipamentos foram permitidos antes das medições. A avaliação do 1RM foi realizada pelo método crescente de cargas. A força máxima estática de handgrip foi considerada como o maior valor desenvolvido em três tentativas de poucos segundos de duração, separadas por um intervalo de 1-2 minutos. A influência da massa muscular sobre a força dos indivíduos foi controlada corrigindo-se os valores da força pela circunferência muscular correspondente (ver medidas antropométricas). Especificamente, as forças do movimento supino, legpress e handgrip foram corrigidas pelas circunferências musculares de braço,

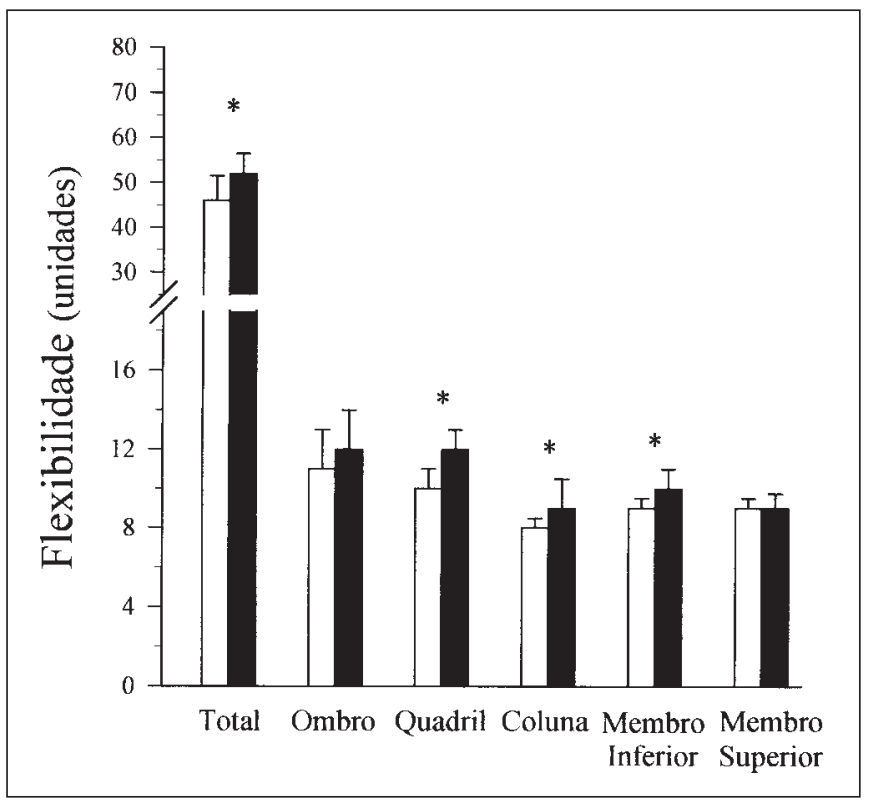

Fig. 1 - Flexibilidade global e segmentar de homens a mulheres $(n=50)$ determinadas pelo Flexiteste; $* p<0,05$ para comparação entre os sexos.

coxa e antebraço. O somatório dos valores individuais de cada movimento foi utilizado como indicador da força máxima global.

\section{Estatística}

Os dados foram submetidos a análise univariada para determinar se a distribuição dos dados era ou não gaussiana e permitia a utilização de métodos estatísticos paramétricos. O teste $t$ simples foi utilizado para comparações entre os sexos de flexibilidade global, segmentar (ombro, quadril, coluna, membros superiores e membros inferiores), força muscular e força corrigida. Caso a distribuição de alguma variável não fosse normal ou quando se tratava de variável descontínua (por exemplo, flexíndice), o teste $U$ de Mann-Whitney foi utilizado para tais comparações. O coeficiente de correlação de Pearson foi utilizado para determinar o grau de associação entre duas variáveis. Os dados com distribuição normal são apresentados como média e erro-padrão e os dados de variáveis descontínuas ou aqueles com distribuição não-paramétrica são apresentados como mediana e amplitude.

\section{RESULTADOS}

A flexibilidade global demonstrou ser maior nas mulheres [flexíndice $=52$ (38-69)] em relação aos homens [flexíndice $=46$ (37-57); $\mathrm{p}=0,004$; figura 1]. Quando analisada segmentarmente, a flexibilidade foi significativamente maior nas mulheres nos movimentos de quadril $(p=0,004)$, coluna ( $p$ $=0,006)$ e membros inferiores $(p=0,011)$. Porém, nos movimentos referentes ao ombro $(\mathrm{p}=0,277)$ e membros superiores $(p=0,067)$, essa diferença não demonstrou ser significativa (figura 1). 


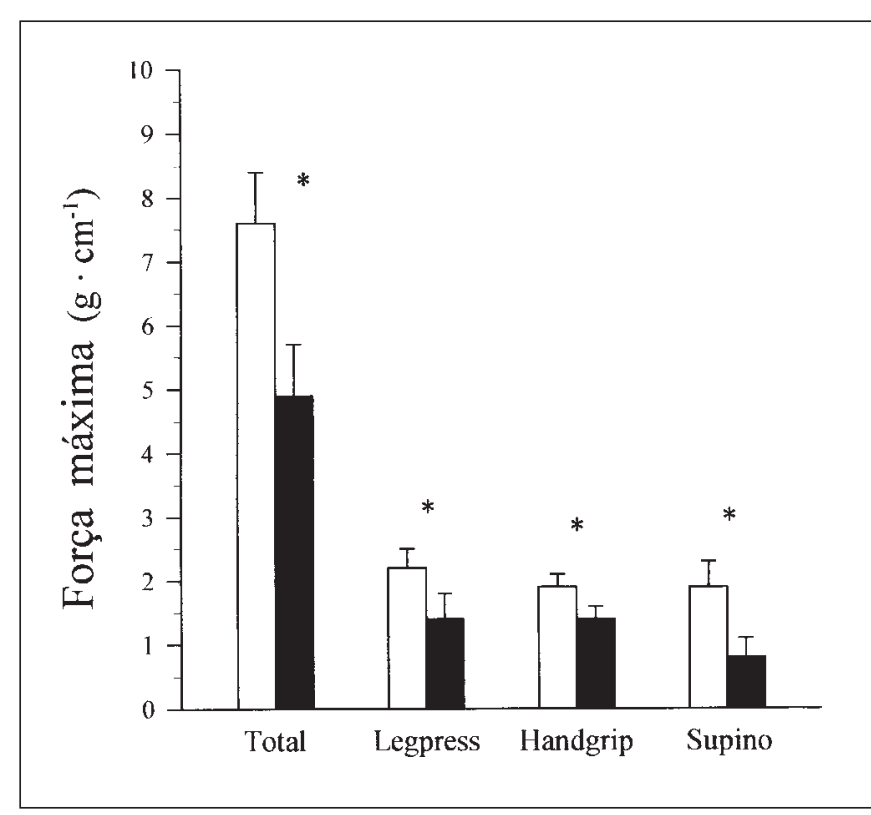

Fig. 2 - Força máxima global e segmentar de homens a mulheres $(n=50)$ determinadas pelo método de $1 R M$ (legpress e supino) e dinamometria (handgrip). ${ }^{*} p<0,05$ para comparação entre os sexos.

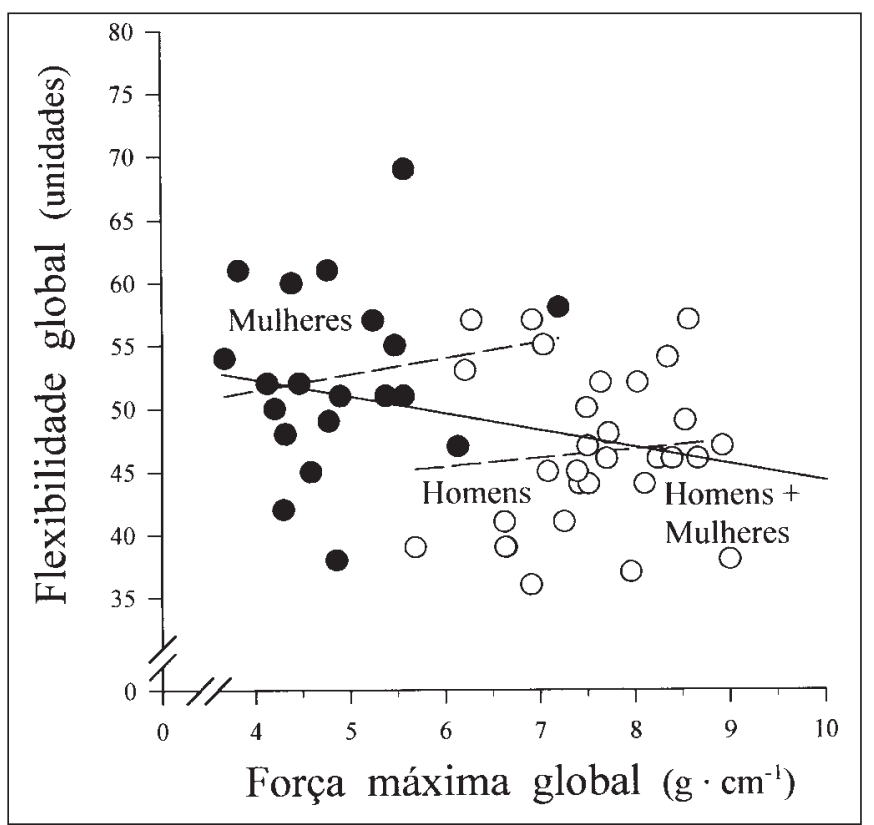

Fig. 3 - Valores individuais de flexibilidade e força máxima globais de homens $(\mathrm{V})$ e mulheres (v). A linha de regressão linear para todos os dados (linha contínua) mostra correlação inversa significativa $(r=-0,297 ; p=$ 0,036 ). As correlações obtidas com os dados separados por sexo (linhas interrompidas) não foram significativas (mulheres: $r=0,149 ; p=0,531$; homens: $r=0,092 ; p=0,628$ ).

A força muscular máxima global foi superior nos homens quando comparada com a das mulheres ( $p=0,001$; figura 2 ). Essa diferença existiu também de forma isolada para os três movimentos estudados ( $\mathrm{p}=0,001$; figura 2$)$.

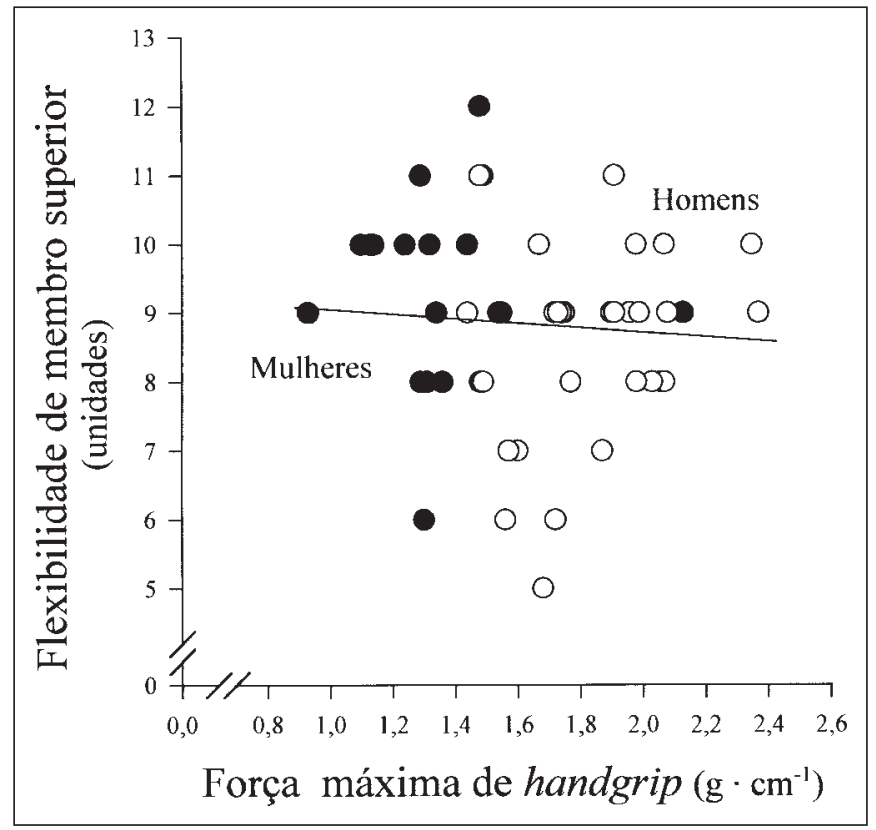

Fig. 4 - Valores individuais de flexibilidade de membro superior e força máxima de handgrip de homens (V) e de mulheres (v). A linha demonstra a ausência de correlação entre as variáveis (homens: $r=0,254 ; p=0,175$; mulheres: $r=-0,088 ; p=0,705)$.

A análise de regressão linear entre flexibilidade global e força muscular máxima global apresentou correlação negativa $(r=-0,297 ; p=0,036)$ quando foram aglutinados os resultados de ambos os sexos. Porém, quando essa análise foi desenvolvida separando-se os resultados quanto ao sexo, não foram observadas correlações significativas (mulheres: $\mathrm{r}=$ 0,$149 ; \mathrm{p}=0,531$; homens: $\mathrm{r}=0,092 ; \mathrm{p}=0,628$; figura 3).

Quando a flexibilidade e a força muscular foram analisados segmentarmente, ou seja, agrupando-se os resultados de movimentos relacionados, não houve nenhuma correlação linear significativa [membro superior feminino vs. handgrip: $\mathrm{r}=-0,088 ; \mathrm{p}=0,705$; membro superior masculino vs. handgrip: $\mathrm{r}=0,254 ; \mathrm{p}=0,175$ (figura 4); ombro feminino $v$ s. supino: $\mathrm{r}=0,063 ; \mathrm{p}=0,787$; ombro masculino $v$ s. supino: $\mathrm{r}$ $=0,136 ; p=0,469$ (figura 5); quadril feminino vs. legpress: $r=0,232 ; p=0,318$; quadril masculino vs. legpress: $r=0,023$; $\mathrm{p}=0,903$ (figura 6)].

\section{DISCUSSÃO}

O presente estudo investigou transversalmente a relação entre flexibilidade e força muscular em indivíduos jovens sadios realizando uma análise global e multiarticular. Essa abordagem metodológica foi possível pela utilização do Flexiteste, que classifica em escala ordinal a amplitude do arco de 20 movimentos articulares diferentes realizados passivamente. Esta característica difere o Flexiteste de outros métodos tradicionais como o sit-and-reach ${ }^{3}$, cujos resultados avaliam apenas um movimento corporal, além de depender do 


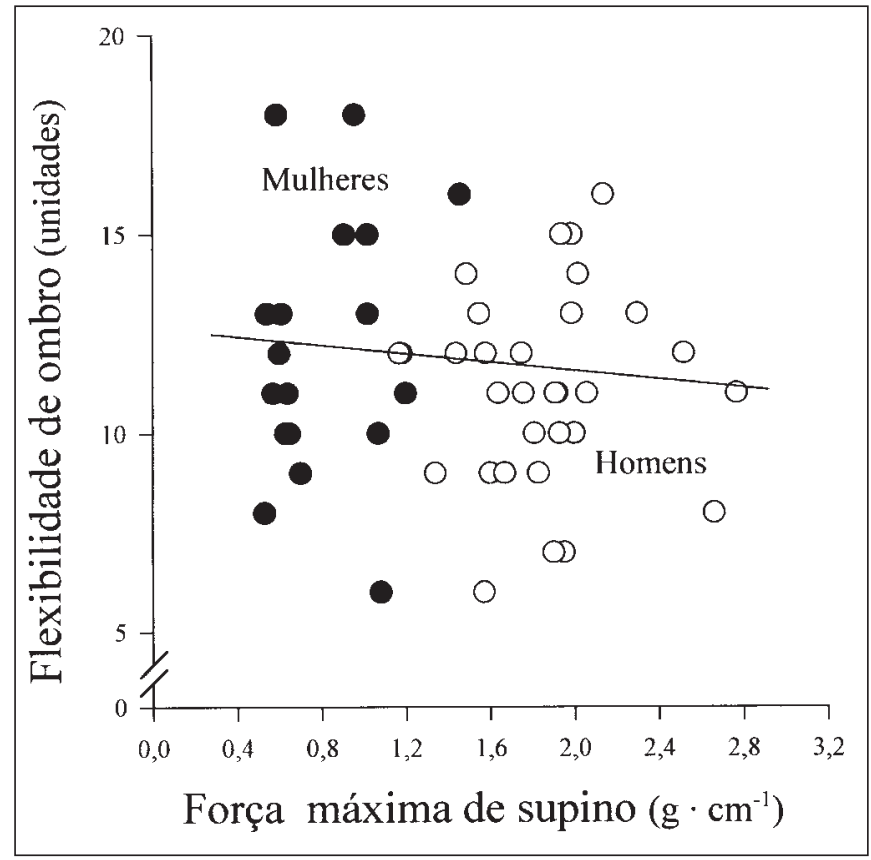

Fig. 5 - Valores individuais de flexibilidade de ombro e força máxima de desenvolvimento supino de homens (V) e de mulheres (v). A linha demonstra a ausência de correlação (homens: $r=0,136 ; p=0,469$; mulheres: $r=$ $0,063 ; p=0,787)$.

comprimento proporcional de membros inferiores e da força para executá-lo ativamente pelo avaliado.

Os resultados mostraram que a força muscular máxima é maior nos homens do que nas mulheres, independente dos grupos musculares avaliados. Por outro lado, em relação à flexibilidade, as mulheres têm, de forma global, maior flexibilidade do que os homens, dados estes que corroboram a literatura ${ }^{3,4}$. Uma contribuição original do presente estudo é a demonstração de que a diferença entre mulheres e homens quanto à flexibilidade localiza-se nos movimentos do quadril, coluna e membro inferior, mas não existe nos movimentos de membro superior e ombro.

Outro aspecto importante é a demonstração da inexistência de correlação entre flexibilidade e força muscular, sugerindo independência entre essas qualidades físicas. Análises de correlação separadas foram realizadas entre força e flexibilidade de homens e mulheres, pois a aglutinação dos resultados de ambos os sexos pode revelar equivocadamente um coeficiente de correlação significativo, conforme exemplificado na figura 3. Os dados obtidos em homens e mulheres têm diferenças sistemáticas de flexibilidade e de força e, por essa razão, devem ser analisados separadamente ${ }^{20}$.

Essa independência entre flexibilidade e força muscular sustenta conceitos atuais ${ }^{4}$ e sugere que o treinamento específico de cada valência não deva interferir mutuamente. Essa questão poderá ser melhor investigada por meio de um protocolo longitudinal de treinamento isolado e combinado de força muscular e flexibilidade. Outros estudos envolvendo

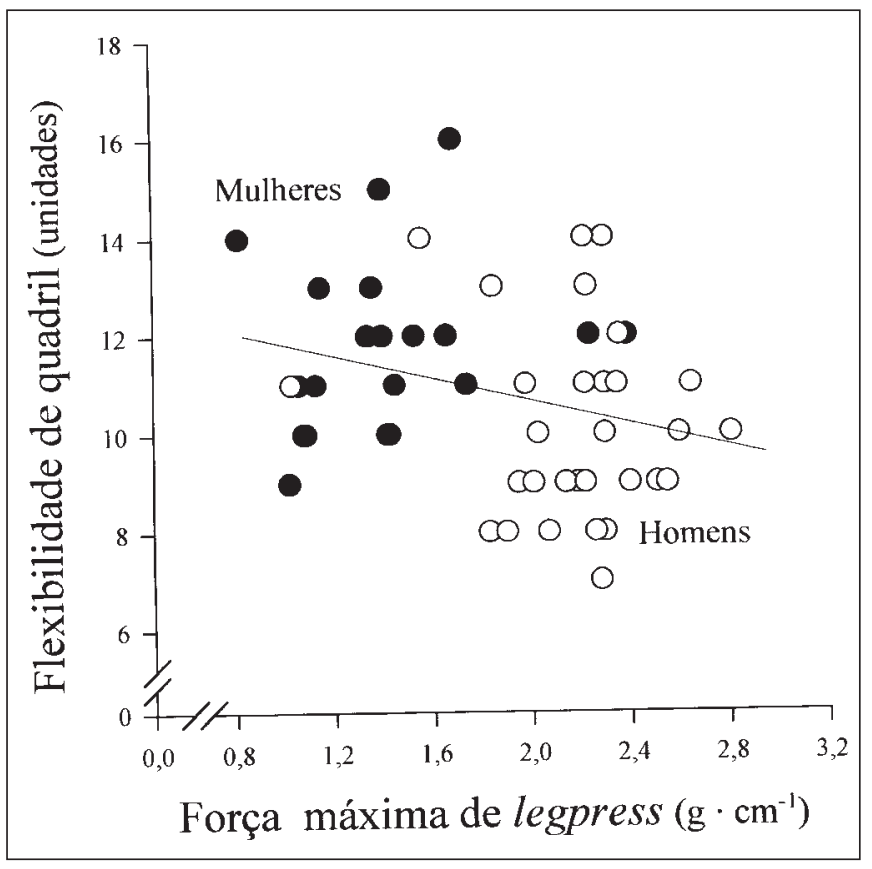

Fig. 6 - Valores individuais de flexibilidade de quadril e força máxima de legpress de homens (V) e de mulheres (v). A linha mostra correlação não significativa (homens: $r=0,023 ; p=0,903$; mulheres: $r=0,232 ; p=0,318$ ).

indivíduos de idades diferentes e portadores de doenças deverão confirmar ou não esses achados obtidos em jovens sadios.

Em conclusão, considerando as características do grupo estudado, os resultados demonstraram que mulheres têm maior flexibilidade que homens, principalmente nos movimentos de coluna, quadril e membros inferiores, enquanto os homens apresentam maior força muscular global e segmentar, mesmo corrigindo-se a diferença de massa muscular. Os resultados sugerem que não existe relação entre força muscular e flexibilidade em adultos jovens sadios.

\section{AGRADECIMENTOS}

Este estudo foi fomentado pelo CNPq (no 20660/95-1).

\section{REFERÊNCIAS}

1. American College of Sports Medicine. ACSM's guidelines for exercise testing and prescription. $5^{\underline{a}}$ ed., Baltimore: Williams \& Wilkins, 1995: 373.

2. Carvalho T, Nóbrega ACL, Lazzoli JK, Magni JRT, Rezende L, Drummond FA, et al. Posição oficial da Sociedade Brasileira de Medicina do Esporte: atividade física e saúde. Rev Bras Med Esport 1996;2:79-81.

3. Hubley-Kozey CL. Testing flexibility. In: MacDougall JC, Wenger HA, Green HJ (eds.) Physiological testing of the high-performance athlete. $2^{\underline{a}}$ ed., Champaign: Human Kinetics, 1990:309-59.

4. McArdle WD, Katch FI, Katch VL. Exercise Physiology - Energy, nutrition, and human performance. $3 \underline{a}$ ed., Philadelphia: Lea \& Febiger, 1991:452-96. 
5. Pollock ML, Wilmore JH. Exercise in health and disease - Evaluation and prescription for prevention and rehabilitation. $3 \underline{a}$ ed., Philadelphia: WB Saunders, 1990.

6. Gardner GW. Effect of isometric and isotonic exercise on joint motion Arch Phys Med Rehab 1966;47:24-30.

7. Girouard CK, Hurley BF. Does strength training inhibit gains in range of motion from flexibility training in older adults? Med Sci Sports Exerc 1995;27:1444-9.

8. Mills EM. The effect of low-intensity aerobic exercise on muscle strength, flexibility, and balance among sedentary elderly persons. Nurs Res 1994; 43:207-11.

9. Jarié S, Ropret R, Kukolj M, Ilié DB. Role of agonist and antagonist muscle strength in performance of rapid movements. Eur J Appl Physiol 1995;71:464-8.

10. Turner JG, et al. Sectors affecting on mineral density in high school girl. N Z Med J 1992;105:930.

11. Farinatti PTV, Monteiro WD. Fisiologia e avaliação funcional. Vol. 1. Rio de Janeiro: Sprint, 1992:61-77.

12. Maffulli N, King JB, Helms P. Training in élite young athletes (the training of young athletes (TOYA) study): injuries, flexibility and isometric strength. Br J Sports Med 1994;2:123-36.
13. Massey BH, Chaudet NL. Effects of systematic, heavy resistive exercise on range of joint movement in young male adults. Res Quart 1956; 27:41-51.

14. Thomas S, Reading J, Shepard RJ. Revision of the Physical Activity Readiness Questionnaire (PAR-Q). Can J Sport Sci 1992;17:338-45.

15. Harrison GG, et al. Skinfold thickness and measurement technique. In: Lohman T, Roche AF, Martorell R (eds.). Anthropometric standardization reference manual. Champaign: Human Kinetics, 1988:55-70.

16. Jelliffe EFP, Jelliffe DB. The arm circumference as a public health index of protein-calorie malnutrition of early childhood. J Trop Pediatr 1969; 15:179.

17. Ross WD, Hebbelink M, Brown SR, Faulkner RA. Kinanthropometric landmarks and terminology. In: Shepard RJ, Lavalee H (eds.). Fitness assessment. Springfield: Charles C. Thomas, 1978:44-50.

18. Araújo CGS. Medida e avaliação da flexibilidade: da teoria à prática [tese de doutorado]. Rio de Janeiro (RJ): Universidade Federal do Estado do Rio de Janeiro, 1986:366.

19. Sheikh K. Disability scales: assessment of reliability. Arch Phys Med Rehabil 1986;67:245-9.

20. Edwards AL. Factors influencing the magnitude of the correlation coefficient. In: An introduction to linear regression and correlation. $2^{\mathrm{a}}$ ed., New York: W.H. Freeman, 1984:35-47. 